\title{
Laserspray Ionization Using an Atmospheric Solids Analysis Probe for Sample Introduction
}

\author{
Frank Zydel, ${ }^{\text {a Sarah Trimpin, }}{ }^{\mathrm{b}}$ and Charles N. McEwen ${ }^{\mathrm{a}}$ \\ ${ }^{a}$ University of the Sciences in Philadelphia, Philadelphia, Pennsylvania, USA \\ ${ }^{\mathrm{b}}$ Wayne State University, Detroit, Michigan, USA
}

\begin{abstract}
A newly introduced high sensitivity laserspray (LSI) mass spectrometry (MS) method that uses laser ablation of a matrix/analyte mixture at atmospheric pressure (AP) to obtain multiply charged ions from nonvolatile as well as high-mass compounds is now implemented using a simple probe device. The probe used in the LSI approach was originally designed for sample introduction into an AP ionization source using the atmospheric solids analysis probe (ASAP) method. Multiply charged mass spectra of peptides and proteins in 2,5-dihydroxybenzoic acid matrix were readily obtained on two mass spectrometers from different manufacturers with sample introduction using melting point tubes. Here we demonstrate rapid analysis by placing four peptide and protein samples on a single melting point tube. Mass spectra were obtained at high-resolution and using ion mobility spectrometry/MS. (J Am Soc Mass Spectrom 2010, 21, 1889-1892) @ 2010 Published by Elsevier Inc. on behalf of American Society for Mass Spectrometry
\end{abstract}

$\mathrm{T}$ The recently introduced laserspray ionization (LSI) mass spectrometry (MS) technique has advantages similar to ESI MS in mass range extension and improved fragmentation related to multiple charging, as well as the advantages of fast direct analysis and laser ablated spatial resolution associated with matrixassisted laser desorption/ionization (MALDI) [1, 2]. The LSI method has been demonstrated on atmospheric pressure ionization (API) instruments from two MS manufacturers [1-4]. Transmission geometry was used to obtain data for the original LSI publications in the absence of any applied voltage [1-3]. A nitrogen laser beam strikes the backside of the matrix/analyte deposited on the opposite side of a glass microscope slide relative to the laser and near the ion entrance orifice of the mass spectrometer. Previously, transmission geometry AP-MALDI experiments reported only singly charged ions [5-8]. It has been shown not to be necessary for the laser beam to pass through the glass to obtain multiply charged ions of peptides [1,9].

Highly charged ions have also been reported using an IR laser with glycerol containing trifluoroacetic acid as a matrix and using a commercial AP-MALDI source [10]. Low abundance multiply charged ions were observed using reflective geometry only after application of $2500 \mathrm{~V}$ applied to the metal MALDI target plate. Other reports of multiply charged ions using liquid matrices at AP also required applied voltage [11-13].

The atmospheric solids analysis probe (ASAP) method [14, 15] is a simple and convenient means of analyzing samples at atmospheric pressure, frequently

Address reprint requests to Dr. C. N. McEwen, Department of Chemistry and Biochemistry, University of the Sciences in Philadelphia, 600 South 43rd Street, Philadelphia, PA 19194, USA. E-mail: cnmcewen@verizon.net without any sample preparation. The ASAP method is applied to analysis of volatile and semivolatile materials, but was recently shown to be applicable to nonvolatile compounds by using a commercial ESI probe to produce ions of peptides, proteins, and other compounds deposited on the melting point tube by a desorption electrospray ionization (DESI)-like method [16]. The DESI-like configuration required precise alignment of the ESI probe with respect to a melting point tube held in place directly in front of the mass spectrometer (MS) ion entrance orifice by the ASAP assembly. While this method is direct and fast, for proteins, under the conditions employed, considerable metal adduction (sodium) was observed. In analogy, the ASAP probe using LSI conditions with disposable melting point tubes is shown here to be a sensitive and rapid way to ionize peptides and proteins, without significant metal adduction. Because the ASAP probe does not interfere with the normal operation of most ion sources [17], it is possible, using minimal effort, to switch between ESI, APCI, ASAP, DESI, and LSI on a single ion source.

\section{Experimental}

All peptides and proteins as well as 2,5-dihydroxybenzoic acid (2,5-DHB) and solvents were obtained from Sigma Aldrich, St. Louis, MO, USA. The common MALDI dried droplet sample preparation method was used in all experiments [18]. One or $2 \mu \mathrm{L}$ of the matrix/analyte in 1:1 acetonitrile:water was applied onto the exterior of a borosilicate melting point tube $(1.5-1.8 \times 100 \mathrm{~mm}$; Kimble Chase, Vineland, NJ, USA) near the closed end using a pipette.

For the Ion Max source of the Orbitrap Exactive (Thermo Fisher Scientific, Bremen, Germany), the only modification to the commercial ASAP kit (M and $\mathrm{M}$ 
Mass Spec., Hockessin, DE, USA) was to replace the standard ASAP flange with one that allowed the bottom edge of the melting point tube to be $2 \mathrm{~mm}$ distance and directly in front of the ion entrance aperture to the MS (Figure 1). In this arrangement, the laser beam is $90^{\circ}$ relative to the length direction of the melting point tube and directly in-line $\left(\sim 180^{\circ}\right)$ with respect to the opening of the heated ion transfer capillary of the MS. The laser beam is directed at the ion entrance aperture and the melting point tube is positioned so that only a small fraction of the laser beam strikes the glass (some adjustment of the laser can be made without adversely affecting the results). For these experiments, the laser beam was focused to $<100 \mu$ diameter at the melting point tube. In this way, the laser beam impinges the sample without transmitting through the glass melting point tube. The maximum inject time was set at $50 \mathrm{~ms}$ and one acquisition per s provided a mass resolution of 100,000 [FWHH, mass-to-charge $(\mathrm{m} / \mathrm{z})$ 200].

The adjustable ASAP probe allows the melting point tube to be moved across the laser beam over a distance of about $1 \mathrm{~cm}$ (up and down direction in Figure 1) as well as rotated to bring fresh sample into the laser beam's path. A Spectra Physics (Newport, CA, USA) VSL 337 ND-S nitrogen laser with attached focusing lens (120 mm focal length) mounted to the laser using an $x, y$-stage (Edmunds Scientific, Tomawanda, NY, USA) was placed outside the front of the Ion Max source. The glass plate on the front flange of the source was removed so that the laser beam passes through the focusing lens and into the source without obstruction. The laser beam was not attenuated (manufacturer's specification, $300 \mu \mathrm{J}$ pulse energy). The ion transfer tube of the Orbitrap Exactive was heated to $350{ }^{\circ} \mathrm{C}$. All lab occupants wore UV-safe safety goggles (ARGON/KTP; Sperian, Smithfield, RI, USA). For the analysis using the ASAP probe, four peptides and proteins were individually dissolved in water $(0.1 \%$ formic acid $)$ and mixed $1: 1$ with a $5 \mathrm{mg} / \mathrm{mL}$ solution of 2,5-DHB in 1:1 acetonitrile:

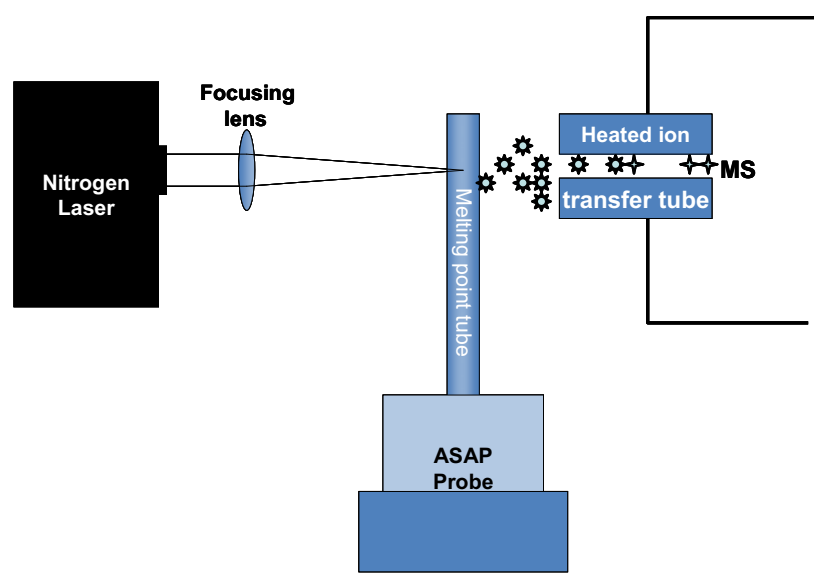

Figure 1. Bottom view representation of the LSI experiment using the ASAP method to introduce samples for analysis on melting point tubes. In the absence of voltage, and using a matrix such as $2,5-\mathrm{DHB}$, multiply charged mass spectra are obtained. water and $2 \mu \mathrm{L}$ placed on a melting point tube at about 2-3 mm intervals. The concentration of each solution was $\sim 1 \mathrm{pm} \mu \mathrm{L}^{-1}$ for angiotensin I and II, $\sim 2.0 \mathrm{pm} \mu \mathrm{L}^{-1}$ for bovine insulin, and $\sim 2.5 \mathrm{pm} \mu \mathrm{L}^{-1}$ for ubiquitin.

The Waters Corporation (Milford, MA, USA) SYNAPT G2 nanospray source was modified as previously described [4]. Briefly, the laser beam from a Spectra Physics nitrogen 337-VSL-ND-S laser was aimed through the opening created by removal of the LockSpray mechanism and directly in-line with the skimmer entrance. The nanospray capillary was also removed so that a melting point tube could be attached to the $x, y, z$-stage normally used to adjust the capillary relative to the ion entrance orifice for ESI. A desolvation device described elsewhere [4] was heated to ca. $250{ }^{\circ} \mathrm{C}$. The melting point tube was positioned $90^{\circ}$ relative to the orifice opening and the laser beam, and within about $2 \mathrm{~mm}$ of the entrance to the homebuilt desolvation device. Sample was applied to only one side of the tube and positioned facing the mass spectrometer ion entrance. The $x, y, z$-stage allowed movement of the melting point tube in the two $90^{\circ}$ directions relative to the laser beam, which in these experiments passed through the melting point tube to strike the matrix/analyte in transmission geometry.

\section{Results and Discussion}

The glass melting point tube transmitted the laser beam so that matrix/analyte applied to the side near the MS ion entrance produced similar results to those with microscope slides in transmission geometry. However, with sample placed over the entire circumference, the laser beam first struck the matrix/analyte on the side opposite the MS entrance and few ions were observed. To be able to deposit the sample over the entire circumference, it was decided to adjust the melting point tube so that only a small part of the laser beam struck the edge (Figure 1). Thus, the laser beam strikes the sample directly without passing through the glass. Rotating or moving the melting point tube $90^{\circ}$ relative to the laser beam exposes fresh sample. Results equivalent in sensitivity and charge distribution to those obtained with microscope slides in transmission geometry were obtained.

The ASAP probe allows the melting point tube to be continuously rotated and moved through the laser beam while acquiring data. Figure $2 a$ shows the selected ion chromatograms for angiotensin I and II, bovine insulin, and ubiquitin in 2,5-DHB matrix applied to a single melting point at $2-3 \mathrm{~mm}$ intervals. The appearance of the ion current profile relates to 'sweet spots' and the movement of the melting point tube relative to the laser beam. Figure $2 b$ shows the mass spectra of all four compounds obtained at 100,000 mass resolution using the Orbitrap Exactive MS. The full range $(m / z 400-2000)$ mass spectra of all compounds were obtained in $<1$ min and carryover between samples was not observed. Adduct ions were not observed for either the peptides or proteins and the LSI method produced improved spectral appearance relative to the 

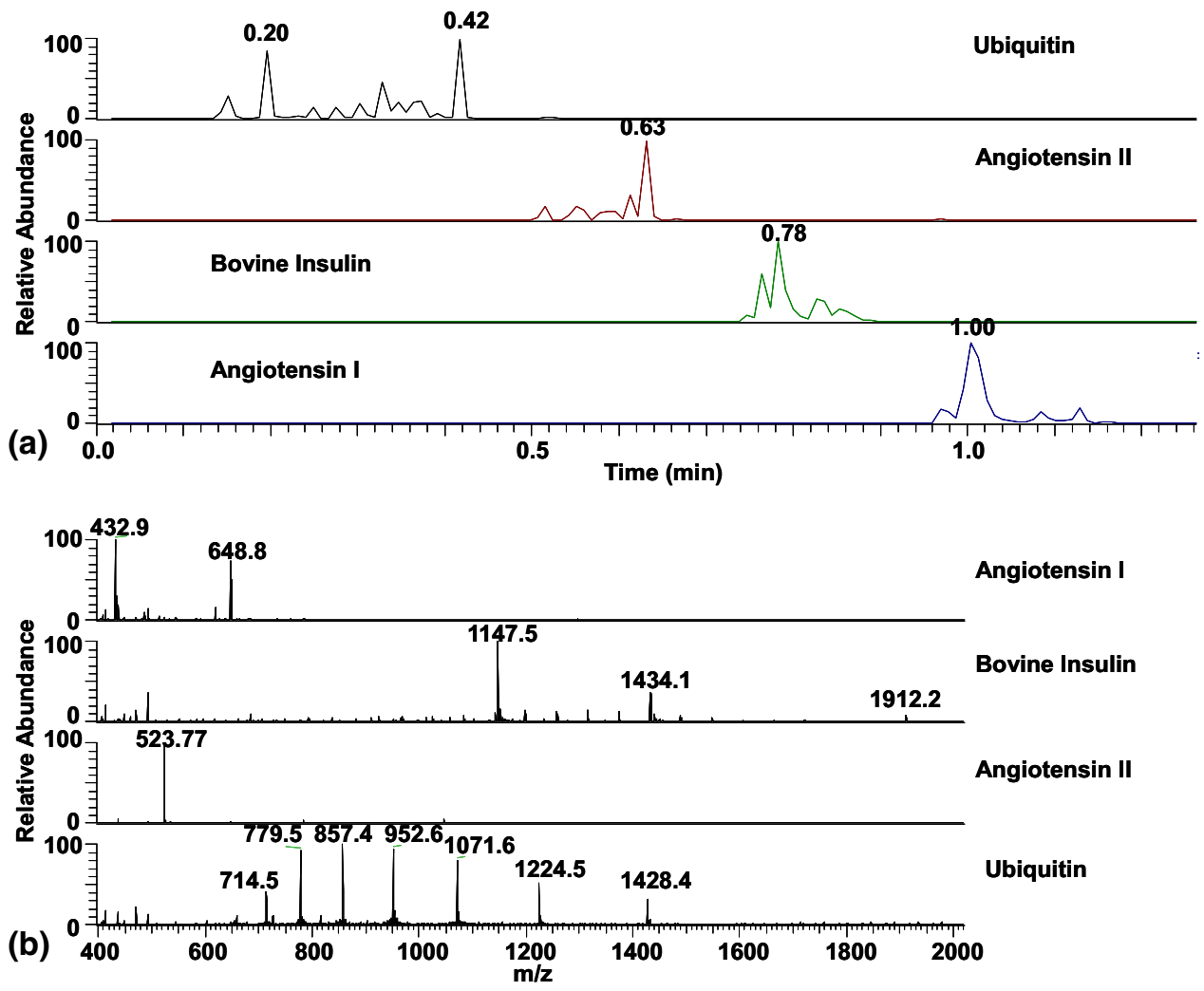

Figure 2. LSI mass spectra of ubiquitin, angiotensin II, bovine insulin, and angiotensin I in 2,5-DHB obtained on a Thermo Fisher Scientific Orbitrap Exactive in $1 \mathrm{~min}$ are shown. The samples were deposited at ca. 2-3 mm intervals on a single melting point tube using the dried droplet method. The melting point tube was moved through the laser beam using the adjustment parameters of the ASAP probe. (a) The selected ion current chromatograms for the $\mathrm{MH}^{+}$ion of each of the four samples. (b) The mass spectra showing the multiply charged mass spectra of the peptides and proteins.

ASAP/DESI-like method [16]. Based on ion abundances, at the concentrations used, it should be possible with faster sample movement to obtain excellent mass spectra for each sample within only a few seconds of acquisition.

The sensitivity of this LSI approach was evaluated by placing $\sim 2$ fmol each of angiotensin I and II in 2,5-DHB matrix on a melting point tube using the dried droplet method and ablating the entire sample. The acquired acquisitions were summed to produce the mass spectrum shown in Figure S1, which can be found in the electronic version of this article. The triply charged ion for angiotensin I (432.90) and the doubly charged ion of angiotensin II (523.77) are readily discerned along with background ions. The multiply charged molecular ions of angiotensin I and II were detectable in a 400 to 2000 mass range acquisition with as little as $300 \mathrm{amol}$ applied to the melting point tube.

Because all background ions are singly charged, ion mobility spectrometry (IMS) MS could theoretically be used to remove the background. Application of IMS-MS to this approach is demonstrated in Figure 3. A melting point tube is used to insert a sample of bovine insulin for LSI analysis on a commercial SYNAPT G2 IMS-MS instrument. The drift time $\left(t_{d}\right)$ versus $m / z$ representation of the data shows the multiply charged insulin ions (labeled) well separated from the low abundance singly charged ions observed at longer drift times. As expected, higher charge state molecular ions drift faster than the lower charge state ions. Sodium adducts are observed but were also observed in the ESI mass spectrum of this sample (data not shown) and was assumed to be a result of storage of the sample in a glass container.

Switching between LSI, ESI, or ASAP MS required less than $2 \mathrm{~min}$, enabling mass spectra of nonpolar vaporizable compounds such as polynuclear aromatic hydrocarbons to be obtained using the ASAP method (data not shown) and nonvolatile compounds such as peptides and proteins by either ESI or LSI within a few min. In a comparison of infusion ESI, using a two-position valve with LSI using the ASAP method for sample introduction, it was found that the LSI method does not show carryover even with high sample concentrations $(50 \mu \mathrm{M})$ so long as the sample does not spread when applying it to the melting point tube. High sample concentrations resulted in carryover issues with infusion ESI.

\section{Conclusion}

An ASAP probe, normally used to insert volatile and semivolatile samples into an AP ion source for analysis, was shown to be a sensitive means of inserting peptides and proteins incorporated in a common MALDI matrix 


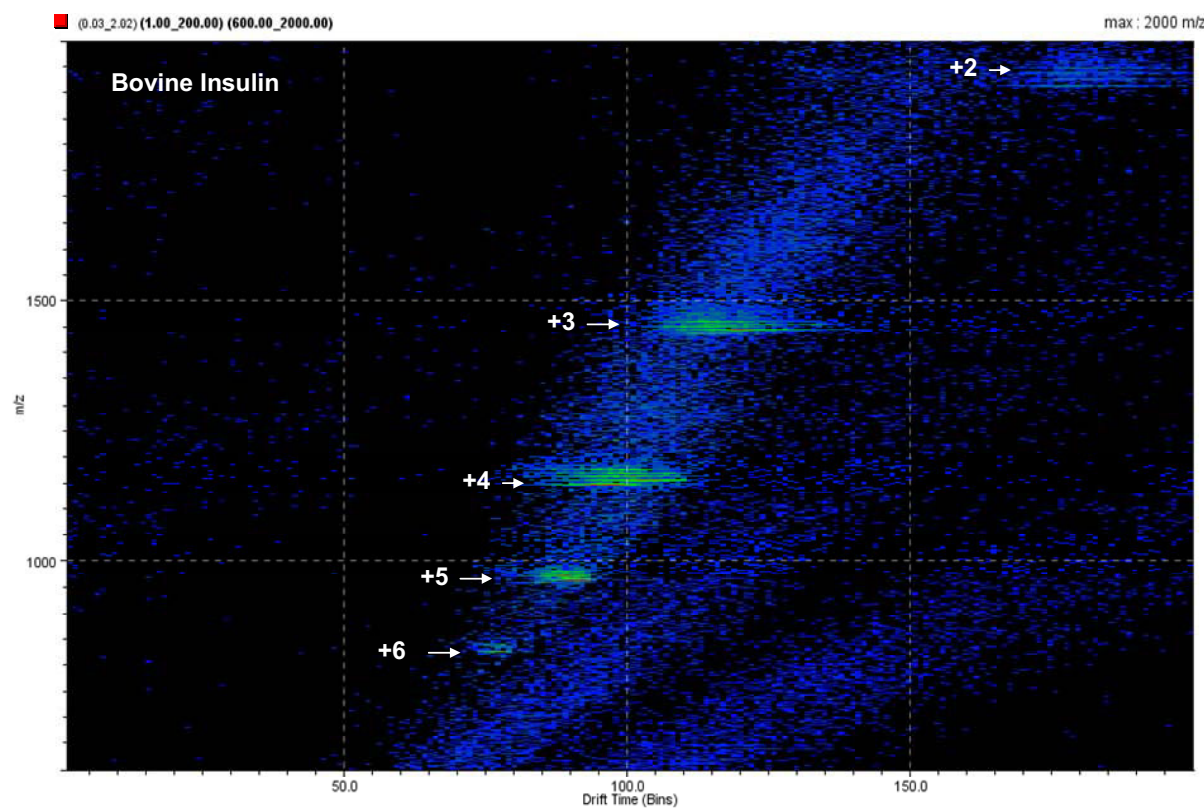

Figure 3. The $t_{d}$ versus $m / z$ DriftScope image of bovine insulin obtained on a Waters SYNAPT G2 mass spectrometer by the LSI method with sample introduction using a melting point tube. Charge states +2 to +6 are denoted by the arrows.

for rapid analysis using the LSI method. The ASAP method had previously been demonstrated to be a fast means for analyzing a wide range of low polarity compounds [14-16]. The combination of LSI and ASAP MS extends the capability of API instruments and offers an easy and forgiving method for analysis of a wide range of materials. IMS-MS readily separates the multiply charged analyte ions produced by LSI from singly charged background ions.

\section{Acknowledgments}

The authors are thankful for the R. E. Houghton grant to the University of the Sciences in Philadelphia (CNM), Wayne State University (ST start up funds), the NSF CAREER award CHE0955975 (ST), and a DuPont Young Investigator Award (ST) for support of this work.

\section{Appendix A Supplementary Material}

Supplementary material associated with this article may be found in the online version at doi:10.1016/ j.jasms.2010.07.005.

\section{References}

1. Trimpin, S.; Inutan, E. D.; Herath, T. N.; McEwen, C. N. Laserspray Ionization-a New AP-MALDI Method for Producing Highly Charged Gas-Phase Ions of Peptides and Proteins Directly from Solid Solutions. Mol. Cell. Proteom. 2010, 9, 362-367.

2. Trimpin, S.; Inutan, E. D.; Herath, T. N.; McEwen, C. N. Matrix-Assisted Laser Desorption/Ionization Mass Spectrometry Method for Selectively Producing Either Singly or Multiply Charged Molecular Ions. Anal. Chem. 2010, 82, 11-15.

3. Trimpin, S.; Herath, T. N.; Inutan, E. D.; Cernat, S. A.; Wager-Miller, J.; Mackie, K.; Walker, J. M. Field-Free Transmission Geometry Atmospheric Pressure Matrix-Assisted Laser Desorption/Ionization of Unadulterated Tissue Samples. Rapid Commun. Mass Spectrom. 2009, 23, 3023-3027.
4. Inutan, E. D.; Trimpin, S. Laserspray Ionization (LSI) Ion Mobility Spectrometry (IMS) Mass spectrometry (MS). J. Am. Soc. Mass Spectrom. 2010, 21, 1260-1264.

5. Galicia, M. C.; Vertes, A.; Callahan, J. H. Atmospheric Pressure MatrixAssisted Laser Desorption/Ionization in Transmission Geometry. Anal. Chem. 2002, 74, 1891-1895.

6. Perez, J.; Petzold, C. J.; Watkins, M. A.; Vaughn, W. E.; Kenttamaa, H. I Laser Desorption in Transmission Geometry Inside a Fourier-Transform Ion Cyclotron Resonance Mass Spectrometer. J. Am. Soc. Mass Spectrom. 1999, 10, 1105-1110.

7. Linnon, I.; J. D.; Glish, G. L. A MALDI Probe for Mass Spectrometers. Anal. Chem. 1997, 69, 2525-2529.

8. Schurenberg, M.; Schulz, T.; Dreisewerd, K.; Hillenkamp, F. MatrixAssisted Laser Desorption/Ionization in Transmission Geometry: Instrumental Implementation and Mechanistic Implications. Rapid Commun. Mass Spectrom. 1996, 10, 1873-1880.

9. McEwen, C. N.; Larsen, B. S.; Trimpin, S. Laserspray Ionization on a Commercial AP-MALDI Mass Spectrometer Ion Source: Selecting Singly or Multiply Charged Ions. Anal. Chem. 2010, 82, 4998-5001.

10. Konig, S.; Kollas, O.; Dreisewerd, K. Generation of Highly Charged Peptide and Protein Ions by Laser Desorption/Ionization Ion Trap Mass Spectrometry. Anal. Chem. 2007, 79, 5484-5488.

11. Sampson, J. S.; Muddiman, D. C. Atmospheric Pressure Infrared (10.6 um) Laser Desorption Electrospray Ionization (IR-LDESI) Coupled to a LTQ Fourier Transform Ion Cyclotron Resonance Mass Spectrometer. Rapid Commun. Mass Spectrom. 2009, 23, 1989-1992.

12. Andrade, F. J.; Shelley, J. T.; Wetzel, W. C.; Webb, M. R.; Gamez, G.; Ray, S. J.; Hieftje, G. M. Atmospheric Pressure Chemical Ionization Source. 1. Ionization of Compounds in the Gas Phase. Anal. Chem. 2008, 80, 2646-2653.

13. Sampson, J. S.; Hawkridge, A. M.; Muddiman, D. C. Development and Characterization of an Ionization Technique for Analysis of Biological Macromolecules: Liquid Matrix-Assisted Laser Desorption Electrospray Ionization. Anal. Chem. 2008, 80, 6773-6778.

14. McEwen, C. N.; McKay, R. G.; Larsen, B. S. Analysis of Solids, Liquids, and Biological Tissues Using Solids Probe Introduction at Atmospheric Pressure on Commercial LC/MS Instruments. Anal. Chem. 2005, 77, 7826-7831.

15. McEwen, C.; Gutteridge, S. Analysis of the Inhibition of the Ergosterol Pathway in Fungi Using the Atmospheric Solids Analysis Probe(ASAP) Method. J. Am. Soc. Mass Spectrom. 2007, 17, 1274-1278.

16. Lloyd, J. A.; Harron, A. F.; McEwen, C. Combination Atmospheric. Pressure Solids Analysis Probe and Desorption Electrospray Ionization Mass Spectrometry Ion Source. Anal. Chem. 2009, 81, 9158-9162.

17. Trimpin, S.; Wijerathne, K.; McEwen, C. N. Rapid Methods of Polymer and Polymer Additives Identification: Multi-Sample Solvent-free MALDI, Pyrolysis at Atmospheric Pressure and Atmospheric Solids Analysis Probe Mass Spectrometry. Anal. Chim. Acta 2009, 654, 20-25.

18. Karas, M.; Hillenkamp, F. Laser Desorption Ionization of Proteins with Molecular Masses Exceeding 10,000 Daltons. Anal. Chem. 1988, 60, 2299-2301. 